BMJ Paediatrics Open

\title{
Using laxatives and/or enemas to accelerate the diagnosis in children presenting with acute abdominal pain: a randomised controlled trial study protocol
}

\author{
Marjolijn E W Timmerman, ${ }^{1}$ Monika Trzpis, ${ }^{2}$ Paul M A Broens ${ }^{1,2}$
}

To cite: Timmerman MEW, Trzpis M, Broens PMA. Using laxatives and/or enemas to accelerate the diagnosis in children presenting with acute abdominal pain: a randomised controlled trial study protocol. BMJ Paediatrics Open 2018;2:e000341. doi:10.1136/ bmjpo-2018-000341

Received 14 July 2018 Revised 29 September 2018 Accepted 4 October 2018

Check for updates

(c) Author(s) (or their employer(s)) 2018. Re-use permitted under CC BY-NC. No commercial re-use. See rights and permissions. Published by BMJ.

${ }^{1}$ Division of Pediatric Surgery, Department of Surgery, University of Groningen, University Medical Center Groningen, Groningen, The Netherlands

${ }^{2}$ Department of Surgery, Anorectal Physiology Laboratory, University of Groningen, University Medical Center Groningen, Groningen, The Netherlands

Correspondence to Marjolijn E W Timmerman; m. timmerman01@umcg.nl

\section{ABSTRACT}

Introduction Many children with acute abdominal pain and suspicion of appendicitis are diagnosed with constipation. Nevertheless, it can be difficult to differentiate between acute constipation and acute appendicitis because of similar symptoms and lack of diagnostic criteria. Consequently, constipation is often missed despite repeated consultations at the emergency department. We hypothesise that the diagnostic process can be improved and adequate treatment accelerated by supporting faecal evacuation in children with acute abdominal pain.

Methods and analysis An unblinded randomised controlled trial including children aged between 5 years and 18 years with acute abdominal pain and suspicion of acute appendicitis. Children who do not have a definitive diagnosis after the first consultation and who need to return for a second consultation will be randomised. The intervention group will receive laxatives and enemas, while the control group will receive no medication. If, after the second consultation, still no diagnosis is established, and a third consultation is needed, then the intervention group will receive only laxatives, and the control group will again not receive medication. The primary outcome will be the differences in abdominal pain scores obtained with FACES Pain Rating Scale and the visual analogue scale at first, second and possibly third consultation. The secondary outcome will be the number of consultations needed to reach final diagnosis.

Ethics and dissemination Laxatives and enemas have proven to be safe and effective treatments for constipation in children. Adverse events are therefore not expected, however, should they occur, then the child concerned shall be properly followed and treated until the event is over. The local Medical Research Ethics Committee approved of this study and waived the otherwise mandatory insurance for human test subjects.

Trial registration numbers Pre-results: CCM0 NL44710.042.12 andEudraCT 2013-000498-56.

\section{INTRODUCTION}

Acute abdominal pain is the third leading cause of visits to emergency departments by children under 15 years and is associated with high costs, especially when hospitalisation is required. ${ }^{1}$ For instance, in the USA, approximately 900000 children visit an emergency department with acute abdominal pain and suspicion of appendicitis annually. ${ }^{2}$ Nevertheless, only $30 \%$ of these children are eventually diagnosed with acute appendicitis, ${ }^{3}$ while constipation is diagnosed in $21 \%$ of girls and $18 \%$ of boys with acute abdominal pain. ${ }^{3}$

It can be difficult to differentiate between constipation with an acute presentation and acute appendicitis, because the symptoms of constipation sometimes mimic those of acute appendicitis. Besides, there are no diagnostic criteria for acute constipation, unlike the Rome IV criteria for diagnosing chronic constipation. ${ }^{4}$ In clinical practice, in order to exclude or confirm constipation, most physicians limit their questioning of patients presenting with acute abdominal pain on whether their bowel habits have changed recently or whether they are 'normal', even though it is known that many people pay scarce attention to their bowel habits and/or are unaware of what 'normal' stool frequencies or consistencies are. ${ }^{56}$ In addition, to diagnose constipation, physicians often avoid performing digital rectal examinations in children because of discomfort or fear on the part of the patients and their own lack of adequate training or experience. ${ }^{7}$ Moreover, additional investigations, such as abdominal X-rays to diagnose children with constipation, were strongly criticised in a number of studies. ${ }^{8-10}$ Because the above-mentioned methods to diagnose constipation are often not performed or carried out insufficiently, an extra consultation the following day can be recommended to determine whether the symptoms improve or worsen. Nonetheless, even after 
extra consultations many children are diagnosed with non-specific abdominal pain instead of constipation. ${ }^{11}$ These misdiagnoses could result in chronic abdominal pain, because the underlying cause, constipation, is left untreated. ${ }^{12}{ }^{13}$ We believe therefore that a better method is needed to either confirm or exclude constipation in children presenting with acute abdominal pain at the emergency department.

Laxatives and/or enemas are standard treatment for faecal disimpaction in children with constipation. ${ }^{9}$ This combination of medications can provide a rapid relief of symptoms and has proven to be safe, and adverse events rarely occur. ${ }^{9}{ }^{14-16}$ We hypothesise that by supporting faecal evacuation with laxatives and enemas in children suffering from acute abdominal pain but who were not diagnosed after first consultation, the diagnostic process could be accelerated. In case a child does indeed suffer from constipation, laxatives and/or enemas lead to rapid reduction of pain. In addition to the positive effect this has on the child's condition, the reduction of pain also points towards constipation as the cause of the abdominal pain. If a child does not suffer from constipation, then laxatives and/or enemas will not provide significant relief of symptoms, indicating to the physician that constipation is less likely to be the cause of the abdominal pain.

Our objective with this randomised controlled trial is to study whether supporting faecal evacuation in children with acute abdominal pain, but without a definitive diagnosis after the first consultation at the emergency department, could accelerate and improve the diagnostic process.

\section{METHODS AND ANALYSIS}

\section{Study design and setting}

An unblinded randomised controlled trial at the emergency department of an academic hospital, the University Medical Center Groningen, in the Netherlands. This protocol describes a study which can be performed as a pilot study and as a final study with an increased sample size. The pilot study will allow to gain data required to perform power analysis, and in this way will allow the performance of adequately powered randomised follow-up study. The final study is required to evaluate our hypothesis and reach the goal of this study.

\section{Participants}

We intend to include children between 5 years and 18 years who are referred to the emergency department with acute abdominal pain and suspicion of acute appendicitis. If children do not receive a definitive diagnosis after the first consultation and need to return for a second consultation, then they will be randomised. Exclusion criteria are pregnancy and severe comorbidity like malignancy, recent abdominal surgery or known inflammatory bowel disease.

\section{Recruitment}

Children presenting at the emergency department with suspicion of acute appendicitis will be approached to participate in the study. First, the researcher will explain the study protocol to the parent(s)/legal guardian and the child. Subsequently, we will hand them the study brochure that was adapted so as to be readily understood by children. If, after having had some time to read the brochure and the opportunity to ask questions, the parent(s)/legal guardian and/or the child agree to participate in the study, we will ask them to sign the consent form. Dutch law requires that in case of children younger than 12 years old, only the parent(s)/ legal guardian need to sign the consent form. If children are between 12 and 16 years old, both children and their parent(s)/legal guardian are required to sign the consent form. In case of children older than 16 years, only the children are obliged to sign the consent form. Furthermore, if a child refuses to cooperate during the study, consent for further participation in the research will be withdrawn.

\section{Randomisation}

Children will be randomised using sealed envelopes without any stratification factors. The researcher will randomly pick a sealed envelope from a sealed box located in the emergency room and will open it in the presence of the child and parent(s)/legal guardian. The study is not blinded for the children, parent(s)/legal guardian or for the researcher.

\section{Intervention}

If children do not receive a conclusive diagnosis after the first consultation at the emergency room and need to return for a second consultation, then they will be randomised. After randomisation, the intervention group will receive both laxatives and enemas, while the control group receives no laxatives or enemas. If children need to return for a third consultation, the children in the intervention group will receive only laxatives without an enema, while the children in the control group will again receive no study medication. We decided against prescribing enemas to children in the intervention group twice, because we expect a reduced effect after a second time and adverse events may occur.

The dosage of laxatives and enemas will be adjusted according to the children's ages. Children between 5 years and 10 years will receive $4 \mathrm{~g}$ macrogol 4000 twice, while children older than 10 years will receive $10 \mathrm{~g}$ macrogol 4000 twice. In addition, children between 5 years and 12 years will receive a $10 \mathrm{~mL}$ sorbitol enema, while children older than 12 years will receive a $133 \mathrm{~mL}$ sodium phosphate enema. As a safety precaution, children under the age of 12 years will receive the enema in hospital. The laxatives and sodium phosphate enemas for children older than 12 years can be administered at home. 
Table 1 Study schedule

Time points

\begin{tabular}{|c|c|c|c|c|}
\hline & First consultation at ED & $\begin{array}{l}\text { Second consultation } \\
\text { at ED }\end{array}$ & $\begin{array}{l}\text { Third consultation at } \\
\text { ED }\end{array}$ & Close-out \\
\hline Enrolment & $\begin{array}{l}\text { Standard diagnostic procedures. } \\
\text { Eligibility screening. } \\
\text { Informed consent. } \\
\text { Allocation when diagnosis is unknown. }\end{array}$ & & & \\
\hline \multirow[t]{2}{*}{ Intervention } & $\begin{array}{l}\text { Intervention group: laxatives and } \\
\text { enema. }\end{array}$ & $\begin{array}{l}\text { Intervention group: } \\
\text { only laxatives. }\end{array}$ & & \\
\hline & Control group: no study medication. & $\begin{array}{l}\text { Control group: no } \\
\text { study medication. }\end{array}$ & & \\
\hline \multirow[t]{3}{*}{ Assessments } & Patient characteristics. & & & \\
\hline & $\begin{array}{l}\text { Pain score, used medication and stool } \\
\text { production. }\end{array}$ & $\begin{array}{l}\text { Pain score, used } \\
\text { medication and stool } \\
\text { production. }\end{array}$ & $\begin{array}{l}\text { Pain score, used } \\
\text { medication and stool } \\
\text { production. }\end{array}$ & \\
\hline & & & & $\begin{array}{l}\text { Number of } \\
\text { consultations. }\end{array}$ \\
\hline
\end{tabular}

ED, emergency department.

\section{Outcome measures}

The primary outcome of this study will be the differences in the pain scores for abdominal pain as indicated by the child during the first, second and possibly third consultation. Specifically for the pilot study with a small sample size, the primary outcome will be whether there is a diminish in abdominal pain in the consecutive consultation, measured as a binary variable. We will assess the pain scores by using a combination of the Wong-Baker FACES Pain Rating Scale and the visual analogue scale for pain. The secondary outcome will be the time needed to reach the final diagnosis as expressed in the number of consultations. This involves the number of times a physician decides that an extra consultation is needed in combination with the number of times a child returns to hospital with persistent complaints of abdominal pain.

Additional data we will collect are the patients' characteristics, such as age, sex, weight, height, comorbidities and medication use. The dosage and type of painkillers will be analysed and used to correct the pain scores. In addition, we will collect information on stool frequency, stool consistency and other diagnostic criteria for constipation to evaluate the accuracy of the constipation diagnosis. Finally, we will collect information on whether the children took the study medication correctly and whether children produced stool after taking the medication.

\section{Study protocol}

In table 1 , we provide an overview of the study schedule. After arrival at the emergency department, the child and parent(s)/legal guardian will be approached by the researcher, and informed consent for the study will be obtained. During the standard clinical examination, the researcher will collect data on the outcome measures (eg, the pain scores and patient characteristics). If a child receives a diagnosis after the first consultation at the emergency department, the study protocol ends, and the child will receive standard care. The children suffering from acute abdominal pain, without a definitive diagnosis after the first consultation and who need to return for a second consultation, will be randomly assigned to the intervention group or the control group. During the second consultation, we will again collect data on the outcome measures. If a child receives a diagnosis after the second consultation, the study protocol ends and the child will receive standard care. If, after the second consultation, the physician is still unable to establish a diagnosis and requires the child to return for a third consultation, then the child in the intervention group will receive only laxatives to be administered at home. During the third consultation, we will once again collect data on the outcome measures. After this consultation, the study protocol will end for all children, even if they need to come back for extra consultations. Between 2 months and 3 months after the first consultation, the researcher will check the electronic patient file to see whether the child had returned to the emergency department with abdominal pain in the meanwhile.

\section{Sample size}

This trial will be a pilot study, because the necessary data to perform a sample size calculation are not available in the literature. Nevertheless, we propose to set out by including 30 children before possibly expanding the study.

\section{Statistical analyses}

All analyses will be conducted in SPSS V.23.0 for Windows using a per-protocol analysis. The primary outcome, the pain score, is an ordinal variable and will be reported 
as frequencies per points on the scale. The secondary outcome, the number of consultations required to arrive at a final diagnosis, is a non-parametric variable and will be presented as median with range. Other variables to be analysed include the patient characteristics and use of painkillers. Categorical data will be reported as absolute numbers and percentages, parametric continuous data as means with SDs and non-parametric continuous data as medians with ranges.

For the primary outcome, the Mann-Whitney test will be used to analyse the difference the pain scores obtained between the first and second consultation by comparing the intervention group with the control group. An ordinal logistic regression will be used for this comparison to correct for confounding factors, as the use of painkillers. The secondary outcome, the number of consultations required to arrive at a final diagnosis, will also be analysed using the Mann-Whitney test. A p value below 0.05 will be considered statistically significant.

\section{ETHICS AND DISSEMINATION Data management}

All data will be anonymised directly after collection and stored on a secure part of the network drive of University Medical Center Groningen. The hard copies of the informed consent forms will also be stored securely. Only authorised persons will have access to the data. In accordance with Dutch law, the data will be stored for 15 years.

\section{Patient safety}

Laxatives and enemas have proven to be safe and effective treatments for constipation in children. ${ }^{914}$ We therefore do not we expect any adverse events in this study. Nonetheless, should an adverse event occur, then the child concerned shall be followed until the event is over. Depending on the adverse event, follow-up may require additional tests or medical procedures and/or referral to the general physician or a medical specialist. In addition, an annual safety report will be sent to the local Medical Research Ethics Committee of University Medical Center Groningen. As a result of the expected safety level of this study protocol, our local ethics committee waived the otherwise mandatory insurance for human test subjects.

\section{Ethics approval and consent to participate}

All interventions discussed in this study protocol are in accordance with the 1964 Declaration of Helsinki. A written informed consent will be obtained from the parents/legal guardian of eligible children and/or from the children themselves, depending on their age.

\section{DISCUSSION}

Laxatives and enemas have proven to be effective and safe treatments for children with constipation. ${ }^{914}$ Nevertheless, during the inclusion of participants, we noticed a negative attitude of Dutch parents towards such medication for their children, leading to a larger refusal rate $(23 \%)$ on the part of parents than anticipated. In addition, there were some practical problems, especially during the evening and at night, in which many children $(27 \%)$ had already left the hospital before randomisation and/or receiving study medication because they did not want to wait any longer. Also, $37 \%$ of the children from the intervention group did not take their study medication according to protocol. Medication adherence is a familiar issue, also for chronic constipation in which case the adherence rate is around $38 \%$ for the first month. ${ }^{17}$ We therefore decided in advance on a per-protocol analysis instead of an intention-to-treat analysis, so that a possible deviation from the study protocol would not influence the results. We included the first patient in February 2014; however, as a result of the above-mentioned problems, we have only included 10 patients, as originally described in protocol. In the future, however, it is necessary to perform this study in another country where parents have a more positive attitude towards such medication for their children. One might be surprised by the low number of patients presented in this protocol. We would therefore like to emphasise that this protocol has a twofold application; it can be used first as a pilot study in order to gain the data allowing power analysis, and second, it can be used for adequately powered follow-up study. The only difference between the pilot and the final phase of this study will be the number of the included patients.

Nonetheless, we hope that final study performed according to our study protocol will prove that supporting faecal evacuation with laxatives and enemas can accelerate the relief from pain in these children whose acute abdominal pain results from constipation. Moreover, such a relief from pain, on supported evacuation, would afterwards allow the confirmation of the diagnosis of constipation in these children. Consequently, usage of our protocol may even reduce the number of misdiagnosed children with non-specific abdominal pain, who are left untreated for their constipation symptoms. In addition, if we were to recognise acute constipation more often in these children, we may prevent the development of chronic abdominal pain or chronic constipation, ${ }^{12} 13$ both conditions that are associated reduced quality of life. ${ }^{18} 19$

Acknowledgements The authors would like to thank T van Wulfften Palthe, PhD, for correcting the English manuscript.

Contributors MEWT conceptualised and designed the study protocol, acquires data and drafted the manuscript. MT and PMAB conceptualised and designed the study and critically revised the manuscript for important intellectual content. All the authors read and approved the final manuscript.

Funding The authors have not declared a specific grant for this research from any funding agency in the public, commercial or not-for-profit sectors.

Competing interests None declared.

Patient consent Obtained.

Ethics approval The study is approved by the Medical Research Ethics Committee of University Medical Center Groningen (2012/393). 
Provenance and peer review Not commissioned; externally peer reviewed.

Data sharing statement All data are available on reasonable request by the authors.

Open access This is an open access article distributed in accordance with the Creative Commons Attribution Non Commercial (CC BY-NC 4.0) license, which permits others to distribute, remix, adapt, build upon this work non-commercially, and license their derivative works on different terms, provided the original work is properly cited, appropriate credit is given, any changes made indicated, and the use is non-commercial. See: http://creativecommons.org/licenses/by-nc/4.0/.

\section{REFERENCES}

1. National Center for Health Statistics. National Hospital ambulatory medical care survey: 2009 emergency department summary tables. 2012:1-37.

2. Peery AF, Dellon ES, Lund J, et al. Burden of gastrointestinal disease in the United States: 2012 update. Gastroenterology 2012;143:1179-87.

3. Buddingh KT, Wieselmann E, Heineman E, et al. Constipation and nonspecific abdominal pain in teenage girls referred for emergency surgical consultation. J Pediatr Gastroenterol Nutr 2012;54:672-6.

4. Hyams JS, Di Lorenzo C, Saps M, et al. Functional disorders: children and Adolescents. Gastroenterology 2016;150:1456-68.

5. Walter SA, Kjellström L, Nyhlin H, et al. Assessment of normal bowel habits in the general adult population: the Popcol study. Scand $J$ Gastroenterol 2010;45:556-66.

6. Talley NJ, Weaver AL, Zinsmeister AR, et al. Self-reported diarrhea: what does it mean? Am J Gastroenterol 1994:89:1160-4.

7. Orenstein SR, Wald A. Pediatric rectal exam: why, when, and how. Curr Gastroenterol Rep 2016:18:4.

8. Beinvogl B, Sabharwal S, McSweeney M, et al. Are we using abdominal radiographs appropriately in the management of pediatric constipation? J Pediatr 2017;191:179-83.

9. Tabbers MM, DiLorenzo C, Berger MY, et al. Evaluation and treatment of functional constipation in infants and children: evidence-based recommendations from ESPGHAN and NASPGHAN. J Pediatr Gastroenterol Nutr 2014;58:258-74.

10. Berger MY, Tabbers MM, Kurver MJ, et al. Value of abdominal radiography, colonic transit time, and rectal ultrasound scanning in the diagnosis of idiopathic constipation in children: a systematic review. J Pediatr 2012;161:44-50.

11. Thornton GC, Goldacre MJ, Goldacre R, et al. Diagnostic outcomes following childhood non-specific abdominal pain: a record-linkage study. Arch Dis Child 2016;101:305-9.

12. Rey E, Balboa A, Mearin F. Chronic constipation, irritable bowel syndrome with constipation and constipation with pain/discomfort: similarities and differences. Am J Gastroenterol 2014;109:876-84.

13. Chang L, Lembo AJ, Lavins BJ, et al. The impact of abdominal pain on global measures in patients with chronic idiopathic constipation, before and after treatment with linaclotide: a pooled analysis of two randomised, double-blind, placebo-controlled, phase 3 trials Aliment Pharmacol Ther 2014;40:1302-12.

14. Gordon M, MacDonald JK, Parker CE, et al. Osmotic and stimulant laxatives for the management of childhood constipation. Cochrane Database Syst Rev 2016;8:CD009118.

15. Miller MK, Dowd MD, Friesen CA, et al. A randomized trial of enema versus polyethylene glycol 3350 for fecal disimpaction in children presenting to an emergency department. Pediatr Emerg Care 2012;28:115-9.

16. Bekkali NL, van den Berg MM, Dijkgraaf MG, et al. Rectal fecal impaction treatment in childhood constipation: enemas versus high doses oral PEG. Pediatrics 2009;124:e1108-e1115.

17. Steiner SA, Torres MR, Penna FJ, et al. Chronic functional constipation in children: adherence and factors associated with drug treatment. J Pediatr Gastroenterol Nutr 2014;58:598-602.

18. Warschburger $P$, Hänig J, Friedt $M$, et al. Health-related quality of life in children with abdominal pain due to functional or organic gastrointestinal disorders. J Pediatr Psychol 2014;39:45-54.

19. Youssef NN, Langseder AL, Verga BJ, et al. Chronic childhood constipation is associated with impaired quality of life: a casecontrolled study. J Pediatr Gastroenterol Nutr 2005;41:56-60. 